Hanson, H. und R. J. Haschen, Hoppe-Seyler's Z. physiol. Chem. 310, 213 (1958). - 20. NAGed, W., F. WiLlig und F. H. Schmidr, Klin. Wschr. 42, 447 (1964). - 21. Fleisher, G. A., M. Pankow und C. WARnKa, Clin. chim. Acta (Amsterdam) 9, 254 (1964). - 22. Knöpfer, M., Mh. Chem. 30, 31 (1909). - 23. Pesez, M. und A. Petri, Bull. soc. chim. France 1947, 122. - 24. Watt, G. W. und J. D. Chrisp, Analytic. Chem. 24, 2006 (1952). - 25. Thies, H. und G. Kallinich, Biochem. Z. 324, 485 (1953). - 26. Yale, H. L., K. Losee, J. Martius, H. Holsing, F. M. Perry und J. Bernstein, J. Amer. chem. Soc. 75, 1933 (1953). - 27. Losse, G., H.-J. Hebel und CH. KästNer, J. prakt. Chem. 8, 339 (1959). - 28. Kästner, CH., Diplomarbeit, Mat.-Nat. Fak., Halle/Saale, 1959. - 29. Chambers, R. A. und F. H. Carpenter, J. Amer. chem. Soc. 77, 1522 (1955). - 30. Wergin, A., Naturwissenschaften 52, 34 (1965). - 31. Kallos, J.,
D. KaHN und D. Rizok, Canad. J. Biochem. 42, 235 (1965). 32. Kallos, J., E. L. Arthur, D. RizoK und D. Kahn, Canad. J. Biochem. 43, 135 (1965). - 33. Report of the Comission on Enzymes of the International Union of Biochemistry, Pergamon Press, Oxford 1961. - 34. Aebr, H., in: Rauen, H. M. (Hrsg.): „Biochemisches Taschenbuch“, 2. Teil, S. 167, Springer-Verlag, Berlin-Göttingen-Heidelberg-New York 1964. - 35. BeESE, J., W. FARR, E. GRÜNER und R. J. HASChEN, Klin. Wschr. 44, 1049 (1966). - 36. Bamann, E., J. G. HaAs und H. Trappmann, Naturwissenschaften 46, 73 (1959). - 37. LINEWEAvER, H. und D. BuRK, J. Amer. chem. Soc. 56, 658 (1934). - 38. NeTrer, H., Theoretische Biochemie, Springer-Verlag, Berlin-Göttingen-Heidelberg 1959. - 39. Hughes, H. K., Analytic. Chem. 24, 1349 (1952). 40. Richterich, R., Klinische Chemie, S. 52, S. Karger, BaselNew York 1965.

\title{
Zur Immunchemischen Bestimmung von Enzymproteinen der Alkoholdehydrogenase mit Hilfe der Nephelometrie ${ }^{1}$ )
}

\author{
Yon T. O. KLEINE \\ Aus dem Physiologisch-Chemischen Instituț der Universität Hamburg (Direktor: Prof. Dr. J. Kübnau)
}

(Eingegangen am 4. März 1967)

\begin{abstract}
Mit Hilfe von präzipitierenden, auf Alkoholdehydrogenase aus Hefe und Pferdeleber spezifischen Antikörpern wird ein Mikrotest beschrieben, worin die Enzymprotein-Antikörper-Trübung nephelometrisch serienmäßig bestimmt wird. Im Hefehomogenat sowie im Überschuß an Fremdprotein (menschliches Blutserum) kann mit dieser Methode in einem Gesamtvolumen von 0,40 ml 0,3-0,6 $\mu \mathrm{g}$ und mehr Enzymprotein mit einem mittleten Fehler von $\pm 4 \%$ gemessen werden.
\end{abstract}

Precipitating antibodies to alcoholdehydrogenase (Alcohol: NAD oxidoreductase E. C. 1.1.1.1) from yeast and horse liver were used in a microtest for serial measurement of the turbidity caused by the enzyme-protein-antibody-reaction in vitro. With this method and a mean error of $\pm 4 \%, 0,3$ to $0,6 \mu \mathrm{g}$ of enzyme protein and more per $0,40 \mathrm{ml}$ can be measured in yeast homogenate as well as in the presence of excess foreign protein (human blood serum).

Enzyme werden im allgemeinen mit Hilfe ihrer Reaktionsgeschwindigkeit (Aktivität) unter definierten Standardbedingungen gemessen. Hierbei wird die Enzymquantität nur insofern berücksichtigt, als diejenige Enzymmenge bestimmt wird, die $1 \mu \mathrm{Mol}$ Substrat pro Min. unter jenen Bedingungen umsetzt (1). Für die heutige Enzymchemie sind jene Meßbedingungen bei der Bestimmung von allosterisch oder induktiv beeinflußter Enzymaktivität ungenügend: Eine z. B. in einem Gewebehomogenat erhöhte oder verminderte Enzymaktivität kann auf eine quantitative Vermehrung oder Verminderung des Enzymproteins oder aber auf eine allosterische Steigerung oder Hemmung der Enzymaktivität zurückgeführt werden. Beide Phänomene lassen sich durch die Messung der Reaktionsgeschwindigkeit unter Standardbedingungen nicht voneinander unterscheiden.

Mit Hilfe des nephelometrisch gemessenen AntigenAntikörperkomplexes, einer für quantitative Messungen von Proteinen des Blutes (2-9) bereits bewährten

1) Mit Unterstützung der Deutschen Forschungsgemeinschaft.
Methode haben wir versucht, einen Test zur quantitativen Messung des Enzymproteins aufzubauen, mit dem Enzymprotein im Homogenat oder in Gegenwart hoher Fremdproteinkonzentrationen gemessen werden kann. Durch die simultane Messung von Menge und Aktivität eines Enzyms ist es u. E. möglich, genauere Aussagen über dieses Enzym zu machen als es die bisherigen Methoden erlaubten.

\section{Methodik}

Erzeugung von Antikörpern auf Alkoholdehydrogenas $\mathrm{e}^{1}$ ) aus Hefe und Pferdeleber

$2 \mathrm{~kg}$ schweren, männlichen Kaninchen (Neuseeländer Albinos) werden mit eintägigem Intervall aufeinanderfolgend $0,5,1,0,1,5$, 2,0 und 4,0 mg Enzymprotein Hefe-Alkoholdehydrogenase (HADH) bzw. Alkoholdehydrogenase aus Pferdeleber (LADH) ${ }^{2,3}$ )

1) Der Trivialname Alkoholdehydrogenase wird hier gebraucht für das Enzym Alkohol: NAD oxidoteductase EC 1.1.1.1.

2) Bezogen von C. F. Boehringet, Mannheim.

3) Abkürzungen: $\mathrm{HADH}=$ Hefe-Alkoholdehydrogenase; $\mathrm{LADH}$ = Leber-Alkoholdehydrogenase; EDTA = Athylendiamintetraessigsäure. 
i. v. injiziert. Die $\mathrm{HADH}$ wurde vor Applikation gegen $0,15 \mathrm{M}$ $\mathrm{NaCl}, 0,002 \mathrm{M}$ EDTA (mit $\mathrm{KHCO}_{3}$ auf $\mathrm{pH}$ 6,0 eingestellt) bei $4^{\circ}$ dialysiert.

4 Wochen nach der ersten Injektion wird nach zwei weiteren Gaben von HADH ein Teil der Tiere durch Entbluten getötet. Nach Absetzen des Blutkuchens wird das Serum durch niedertouriges Zentrifugieren gewonnen (Antiserum HADH Charge A, Titer 4). Andere Tiere werden wie oben mit HADH behandelt, jedoch nach 4-wöchigem Intervall mit 3 aufeinanderfolgenden i. v.-Gaben von je 1,2 mg Enzymprotein geboostert und 2 Monate nach der ersten Injektion wie oben getötet (Antiserum $H A D H$ Charge $B$, Titer 16). $\mathrm{Da}$ sich auf die oben angegebene Weise keine präzipitierenden Antikörper auf Alkoholdehydrogenase aus Pferdeleber ( $\mathrm{LADH}$ ) erzeugen lassen, wird das LADH-Enzymprotein mit in $0,15 \mathrm{M} \mathrm{NaCl}$ suspendiertem, sterilen $\mathrm{Al}_{2} \mathrm{O}_{3}-\mathrm{Gel}^{1}$ ) gemischt und i. v. appliziert. Damit lassen sich nach dem unter.HADH angegebenen Applikationsmodus 4 Wochen nach Erstinjektion LADH-präzipitierende Antikörper nachweisen. 2 Monate später werden die Tiere entblutet (Antiserum LADH, Titer 8).

\section{Nachweis von präzipitierenden Antikörpern gegen} $\mathrm{HADH}$ und $\mathrm{LADH}$

\section{Oucbterlony-Test}

Der Ouchterlony-Plattentest (10) wird bei Zimmertemperatur in 1 proz. Agar ${ }^{2}$ ) durchgeführt, welcher in Veronalpuffer $\mathrm{pH} 8,6$ $(0,025 \mathrm{M}$ Natrium-diäthylbarbiturat, $0,005 \mathrm{M}$ Diäthylbarbitursäure, $0,025 \mathrm{M}$ Natriumacetat, Ionenstärke $\mu=0,05$ ) oder in $0,05 \mathrm{M}$ Triäthanolaminpuffer $\mathrm{pH}$ 7,4 gelöst war. Zur Titerbestimmung der Antiseren wird eine konstante Menge Antigen (HADH, LADH) gegen $0,03 \mathrm{ml}$ Antiserum aufgetragen, das $1: 1,1: 2,1: 4$ usw. mit $0,15 \mathrm{M} \mathrm{NaCl}$ verdünnt worden war. Bei Angabe des Titers wird diejenige Serumverdünnung angegeben, bei der eine gut sichtbare Präzipitationslinie beobachtet wird.

\section{Immunelektrophorese}

Die Immunelektrophorese nach Grabar (11) und ScheIdegGER (12) wird mit einer LKB-Apparatur (LKB $6800 \mathrm{~A}$ ) in 1 proz. Agar $^{2}$ ) durchgeführt, welcher in Veronalpuffer $\mathrm{pH} \mathrm{8,6} \mathrm{(s.} \mathrm{o.)} \mathrm{oder}$ in 0,05 M Triäthanolaminpuffer $\mathrm{pH} 7,4$ gelöst war. Laufzeit 45-60 Min. bei $250 \mathrm{~V}$ und $4^{\circ}$.

Die Präzipitationslinien werden mit Amidoschwarz 10B (Merck) angefärbt.

\section{EnzJ'maktivitätshemmtest}

Enzymaktivitätshemmtest für $H A D H: 0,2 \mathrm{~m} l \mathrm{HADH}$-Antiserum werden mit $0,2 \mathrm{ml} \mathrm{HADH}-\mathrm{Lösung}(\triangleq 6 \mu \mathrm{g}$ Enzymprotein mit $0,15 \mathrm{M} \mathrm{NaCl}, 0,002 \mathrm{M}$ an EDTA, verdünnt) $120 \mathrm{Min}$. bei $37^{\circ}$ und 23 Stdn. bei $1^{\circ}$ inkubiert.

In gleicher Weise werden folgende Kontrollen angesetzt:

a) 0,2 ml HADH-Lösung (s. o.) mit $0,2 \mathrm{ml}$ Serum vom unbehandelten Kaninchen,

b) $0,2 \mathrm{ml} \mathrm{HADH}$-Antiserum mit $0,2 \mathrm{ml} 0,15 \mathrm{M} \mathrm{NaCl}, 0,002 \mathrm{M}$ an EDTA,

c) $0,2 \mathrm{~m} l$ Serum rom unbehandelten Kaninchen mit 0,2 $\mathrm{m} l 0,15 \mathrm{M}$ $\mathrm{NaCl}, 0,002 \mathrm{M}$ an EDTA.

Verschiedene Zeiten nach Vereinigung von Antiserum und Antigen wird jeweils $0,01 \mathrm{ml}$ aus dem Ansatz entnommen. Die enzymatische Aktivität wird im Photometer Eppendorf bei $334 \mathrm{~nm}$ und $37^{\circ}$ in einer Thermoküvette der Schichtdicke $d=1 \mathrm{~cm}$ gemessen.

Der Ansatz für den UV-Test enthält: $0,01 \mathrm{~m} l$ Probe, $0,02 \mathrm{~m} l 0,1 \mathrm{M}$ $\left.\mathrm{NAD}^{3}\right)$ und 1,97 $\mathrm{m} l$ Puffer-Substrat-Gemisch (250 ml 0,06M $\mathrm{Na}_{4} \mathrm{P}_{2} \mathrm{O}_{7}$-Puffer $\mathrm{pH} 8,5$ und $50 \mathrm{ml} 2 \mathrm{M}$ Äthanol vereinigt) (13).

1) Bezogen von Behringwerke, Marburg.

2) Special Agar Noble, Difco Laboratories, Detroit, USA.

3) Die Testsubstanzen wurden von den Firmen C. F. Boehringer, Mannheim, und E. Merck, Darmstadt, bezogen.
In gleicher Weise wird die Aktivität der Kontrollen bestimmt. Von der jeweils gemessenen Aktivität des Ansatzes bzw. der Kontrolle a) wird die der Kontrollen b) und c) substrahiert.

Eine Enzymeinheit ist diejenige Menge $\mathrm{HADH}$, die unter obigen Bedingungen $1 \mu \mathrm{Mol}$ Athanol pro Min. umsetzt.

Enzymaktivitätshemmtest für $L A D H: 0,1 \mathrm{~m} l \mathrm{LADH}$ (스 $10 \mu \mathrm{g}$

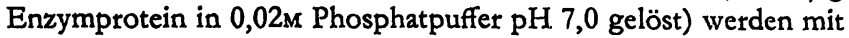
$0,2 \mathrm{ml} \mathrm{LADH}$-Antiserum $120 \mathrm{Min}$. bei $37^{\circ}$ und $24 \mathrm{Stdn}$. bei $1^{\circ}$ inkubiert.

In gleicher Weise werden folgende Kontrollen angesetzt:

a) $0,1 \mathrm{~m} l \mathrm{LADH}$ (s. o.) mit $0,2 \mathrm{~m} l$ Serum vom unbehandelten Kaninchen,

b) $0,2 \mathrm{~m} l \mathrm{LADH}$-Antiserum mit $0,1 \mathrm{~m} l \quad 0,02 \mathrm{M}$ Phosphatpuffer $\mathrm{pH} 7,0$,

c) $0,2 \mathrm{~m} /$ Serum vom unbehandelten Kaninchen mit 0,1 $\mathrm{m} / 0,02 \mathrm{M}$ Phosphatpuffer.

Verschiedene Zeiten nach Vereinigung von Antiserum und Antigen wird jeweils $0,10 \mathrm{~m} l$ aus Ansatz bzw. Kontrollen entnommen und die Enzymaktivität wie oben gemessen.

Der Ansatz für den UV-Test enthält: $0,10 \mathrm{~m} /$ Probe, $0,10 \mathrm{~m} / 0,02 \mathrm{M}$ $\left.\mathrm{NAD}^{3}\right), 0,10 \mathrm{ml}$ Äthanol p. a. und $2,7 \mathrm{ml} 0,1 \mathrm{M}$ Glycin- $\mathrm{NaOH}-$ Puffer pH 9.6 (14). Die Enzymaktivität der Kontrollen b) und c) wird von der jeweiligen Aktivität des Ansatzes bzw. der Kontrolle a) substrahiert. Eine Enzymeinheit ist diejenige Menge LADH, die unter obigen Bedingungen $1 \mu \mathrm{Mol}$ Äthanol pro Min. umsetzt.

\section{Nephelometrische Enzymproteinmessung Aufstellung von Eichleurven für $H A D H$}

Die bei der Vereinigung von Enzymprotein mit spezifischem Antikörper auftretende Trübung wird im Photometer Eppendorf bei $334 \mathrm{~nm}$ in einer Thermoküvette ${ }^{1}$ ) der Schichtdicke $d=1 \mathrm{~cm}$ bei $37^{\circ}$ in folgendem Ansatz gemessen:

$\mathrm{Zu} 0,2 \mathrm{ml}$ Antiserum der Charge A wird die zu $2,0 \mathrm{~m} /$ Gesamtvolumen benötigte Menge an $0,15 \mathrm{M} \mathrm{NaCl}, 0,002 \mathrm{M}$ an EDTA, zugegeben und nach Mischen die Reaktion mit 0,6-27,0 $\mu \mathrm{g} \mathrm{HADH}$ in $0,15 \mathrm{M} \mathrm{NaCl}, 0,002 \mathrm{M}$ an EDTA, gelöst, in der Küvette gestartet. Der Blindwert (Ansatz ohne $\mathrm{HADH}$ ) wird von der jeweiligen Extinktion abgezogen.

$\mathrm{Da}$ die meßbare Extinktionsänderung in einem Gesamtvolumen von $2,0 \mathrm{ml}$ gering und außerdem die Bildung des Antigen-Antikörperkomplexes durch EDTA gehemmt werden kann (15), haben wir das Gesamtvolumen auf $0,35 \mathrm{~m} l$ reduziert und EDTA weggelassen.

In folgendem Ansatz werden 0,2 ml Antiserum Charge B mit 0,15M $\mathrm{NaCl}$ unter Berücksichtigung der zugegebenen $\mathrm{HADH}-\mathrm{Menge}$ $(0,15-9,0 \mu \mathrm{g})$, durch die die Reaktion in der Küvette bei $25^{\circ}$ ausgelöst wird, auf $0,35 \mathrm{~m} l \mathrm{Gesamtvolumen}$ aufgefüllt. Im gleichen Ansatz wird der Einfluß verschiedener $\mathrm{NaCl}$-Konzentrationen $(0,015 \mathrm{M}$ und 1,5M) auf die Bildung des Antigen-Antikörperkomplexes untersucht.

Messung von HADH-Enz ymproteinen in Serum und Hefe-Homogenat

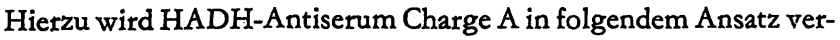
wandt: $\mathrm{Zu} 0,1 \mathrm{ml} \mathrm{HADH}-A n t i s e r u m$ wird die für ein Gesamtvolumen von $0,40 \mathrm{ml}$ erforderliche Menge an $0,15 \mathrm{M} \mathrm{NaCl}$ gegeben und die Reaktion nach Mischen mit 0,15-3,0 $\mu \mathrm{g}$ HADH zur Aufstellung einer Eichkurve oder mit $0,02 \mathrm{ml}$ Hefe-Homogenat in der 1-cm-Küvette bei $25^{\circ}$ ausgelöst und bei $366 \mathrm{~nm}$ gemessen $\left.{ }^{1}\right)$. Die in diesem Ansatz zur Verfügung stehenden $0,3 \mathrm{ml}$ Testvolumen werden für die Messung von HADH-Enzymproteinen in Gegenwart von Fremdproteinen (Blutserum vom Menschen) verwendet. Die Zusammensetzung der Ansätze zur Messung von HADHEnzymproteinen in Serum und Hefe-Homogenat sind aus den Tabellen 1 und 2 zu ersehen.

1) Temperierbarc Küvettenwechselautomatik für 6 Kleinküvetten mit angeschlossenem Schreiber (lineare Extinktionsregistrierung), Rechteckblende 1770 oder Ringblende 1775 des Photometcrs EPPENDORF. 


\section{Aufstellumg won Eicbleurven fïr $L A D H$}

Die nephelometrische Enzymproteinmessung von LADH erfolgt bis $334 \mathrm{~nm}$ und $25^{\circ}$ nach dem gleichen Prinzip im folgenden Ansatz:

$\mathrm{Zu} 0,2 \mathrm{~m} / \mathrm{LADH}$ Antiserum wird die für ein Gesamtvolumen von $0,35 \mathrm{ml}$ erforderliche Menge an $0,15 \mathrm{M} \mathrm{NaCl}$ gegeben und die Reaktion mit $0,25-6 \mu \mathrm{g} \mathrm{LADH}$ in der $1-\mathrm{cm}-$ Küvette ausgelöst $\left.{ }^{1}\right)$. Im gleichen Ansatz wird der Einfluß verschiedener $\mathrm{NaCl}-$ Konzentrationen (0,015 $\mathrm{M}$ und $1,5 \mathrm{M})$ auf die Bildung des Antigen-Antikörperkomplexes untersucht.

\section{Leerwert}

$\mathrm{Da}$ die Extinktion des Leerwertes (Ansatz ohne Antigen) trotz Zentrifugierens der verwendeten Lösungen in einem Gesamtvolumen von $0,35 \mathrm{bzw} .0,40 \mathrm{ml}$ für $0,2 \mathrm{bzw} .0,1 \mathrm{~m} /$ hämolysefreies

Tab. 1

Nephelometrische Messung des Enzymproteins von Alkoholdehydrogenase in Hefehomogenat $\left(28 \mathrm{IU}\left(37^{\circ}\right) / \mathrm{mg}\right.$ Biuretprotein). Eingesetzt werden $0,02 \mathrm{ml}$ Homogenat $\hat{=} 0,145 \mathrm{IU} \hat{=} 0,69 \mu \mathrm{g}$ Enzymprotein des Eichpräparates. Gesamtvolumen $0,40 \overline{\mathrm{m} l}, 366 \mathrm{~nm}, \mathrm{~d}=$ $1 \mathrm{~cm}, 25^{\circ}$. Die Reaktion wird in der Küvette mit 0,02 $\mathrm{ml}$ Homogenat ausgelöst. Doppelbestimmung

\begin{tabular}{|c|c|c|}
\hline & $\begin{array}{c}E_{38 \%} \\
\text { nach } 50 \text { Min. } \\
\text { Inkubation }\end{array}$ & $\begin{array}{c}E_{388} \\
\text { nach } 70 \text { Min. } \\
\text { Inkubation }\end{array}$ \\
\hline \multicolumn{3}{|c|}{ Eingesetzt: $0,69 \mu$ Enzymprotein (rechnerisch ermittelt) } \\
\hline $\begin{aligned} \text { Leerwert a): } & \begin{array}{l}0,10 \mathrm{ml} \text { Antiserum Charge A } \\
0,30 \mathrm{~m} l \quad 0,15 \mathrm{M} \mathrm{NaCl}\end{array}\end{aligned}$ & 0,013 & 0,018 \\
\hline $\begin{aligned} \text { Leerwert b) }: & 0,02 \mathrm{~m} l \text { Homogenat } \\
0,38 \mathrm{~m} l & \text { 0,15M NaCl }\end{aligned}$ & 0,014 & 0,017 \\
\hline $\begin{array}{l}0,10 \mathrm{ml} \text { Antiserum Charge A } \\
0,28 \mathrm{ml} 0,15 \mathrm{M} \mathrm{NaCl} \\
0,02 \mathrm{ml} \text { Homogenat }\end{array}$ & 0,093 & 0,101 \\
\hline Probe - Leerwerte a) und b): & 0,066 & 0,066 \\
\hline \multicolumn{3}{|c|}{ Gefunden: 0,82 bzw. $0,73 \mu \mathrm{g}$ Enzymprotein } \\
\hline
\end{tabular}

Tab. 2

Nephelometrische Messung des Enzymproteins der Hefealkoholdehydrogenase (HADH) in Blutserum vom Menschen. Gesamtvolumen $0,40 \mathrm{ml}, 366 \mathrm{~nm}, \mathrm{~d}=1 \mathrm{~cm}, 25^{\circ}$. Inkubationszeit 50 Min. Die Reaktion wird mit $0,10 \mathrm{ml}$ Antiserum in der Küvette ausgelöst

\begin{tabular}{|c|c|c|c|}
\hline & & $E^{300} \mathrm{~nm}$ & \\
\hline Eingesetzt: & \multicolumn{3}{|l|}{$0,3 \mu \mathrm{g}$ HADH-Enzymprotein } \\
\hline Leerwert a): & $\begin{array}{l}0,10 \mathrm{ml} \text { Antiserum Charge A } \\
0,20 \mathrm{ml} \text { Blutserum yom Menschen } \\
0,10 \mathrm{ml} 0,15 \mathrm{M} \mathrm{NaCl}\end{array}$ & 0,104 & \\
\hline Leerwert b): & $\begin{array}{l}\text { 0,01 } \mathrm{ml} \text { HADH } \\
0,20 \mathrm{ml} \text { Blutserum vom Menschen } \\
0,19 \mathrm{ml} 0,15 \mathrm{M} \mathrm{NaCl}\end{array}$ & 0,022 & \\
\hline Probe: & $\begin{array}{l}0,01 \mathrm{ml} \text { HADH } \\
0,09 \mathrm{ml} 0,15 \mathrm{M} \text { NaCl } \\
0,20 \mathrm{ml} \text { Blutserum vom Menschen } \\
0,10 \mathrm{ml} \text { Antiserum Charge A }\end{array}$ & 0,149 & \\
\hline \multicolumn{2}{|c|}{ Probe - Leerwert a) und b) } & 0,023 & \\
\hline gefunden: & $0,29 \mu \mathrm{g}$ HADH-Enzymprotein & & \\
\hline Eingesetzt: & $0,6 \mu \mathrm{g}$ HADH-Enzymprotein & & \\
\hline Leerwert: & $\begin{array}{l}0,02 \mathrm{ml} \text { HADH } \\
0,20 \mathrm{ml} \text { Blutserum vom Menschen } \\
0,08 \mathrm{ml} 0,15 \mathrm{M} \text { NaCl } \\
0,10 \mathrm{ml} \text { Kaninchennormalserum }\end{array}$ & $\underset{0,080}{A}$ & $\begin{array}{c}\mathrm{B} \\
0,012\end{array}$ \\
\hline Probe: & $\begin{array}{l}0,02 \mathrm{ml} \text { HADH } \\
0,20 \mathrm{ml} \text { Blutserum vom Menschen } \\
0,08 \mathrm{ml} 0,15 \mathrm{M} \mathrm{NaCl} \\
0,10 \mathrm{~m} l \text { Antiserum Charge A }\end{array}$ & A bzw. B & 0,059 \\
\hline \multicolumn{2}{|c|}{ Probe - Leerwert: } & 0,051 & 0,047 \\
\hline
\end{tabular}

gefunden: 0,64 bzw. $0,59 \mu$ g HADH-Enzymprotein

1) Temperierbare Küvettenwechselautomatik für 6 Kleinküvetten mit angeschlossenem Schreiber (lineare Extinktionsregistrierung), Rechteckblende 1770 oder Ringblende 1775 des Photometers EPPENDORF,
Antiserum bei einer Inkubationstemperatur von $37^{\circ}$ steil ansteigt, wird diese auf $25^{\circ}$ herabgesetzt und die bei dieser Temperatur verlangsamte Antigen-Antikörperreaktion in Kauf genommen. Weitere Versuche, die Extinktion des Leerwertes zu erniedrigen (Filtrieren, Inkubation bei $56^{\circ}$ mit anschließendem Filtrieren, Ultrazentrifugation des Antiserums), zeitigten nicht den gewünschten Erfolg.

\section{Ergebnisse}

Erzeugung und Nachweis von Antikörpern Alkoboldebydrogenase aus Hefe ( $H A D H)$

8 Tage nach der ersten Injektion lassen sich immunelektrophoretisch und im Ouchterlony-Test bereits HADH-
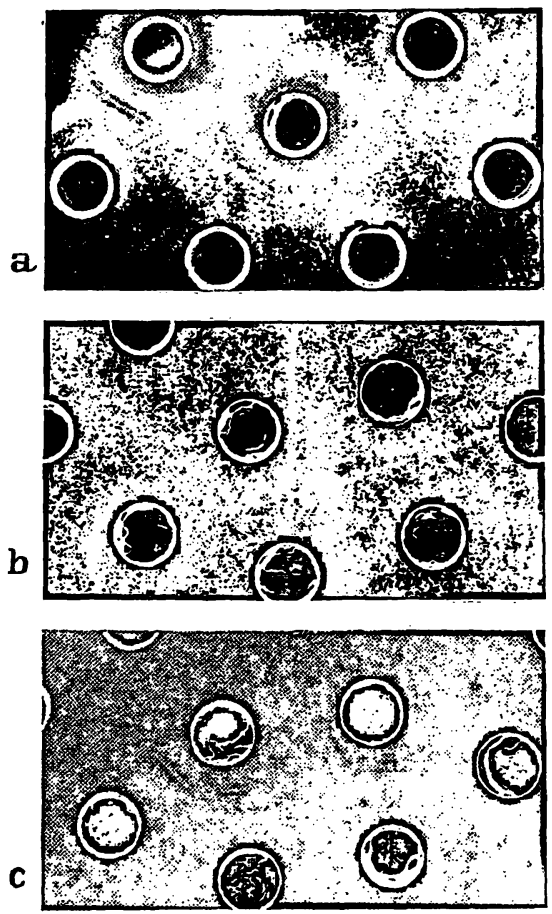

Abb. 1

Oucbterlony-Platten-Test in 1 proz. Agar in Veronalpuffer $\mathrm{pH} \mathrm{8,6}$ Obere Löcher: Antigen: a) und b) Hefealkoholdehydrogenase (HADH), c) Leberalkoholdehydrogenase (LADH)

Untere Löcher: Antiserum: a) HADH-Antiserum Charge A, b) HADHAntiserum Charge $B, c)$ LADH-Antiserum Einzelheiten s. Text

a

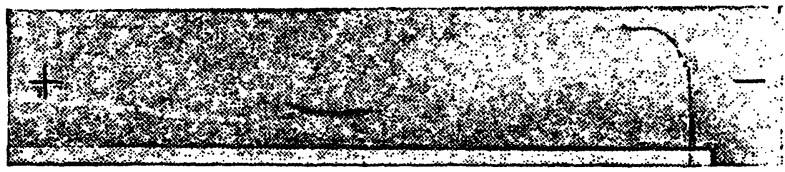

b
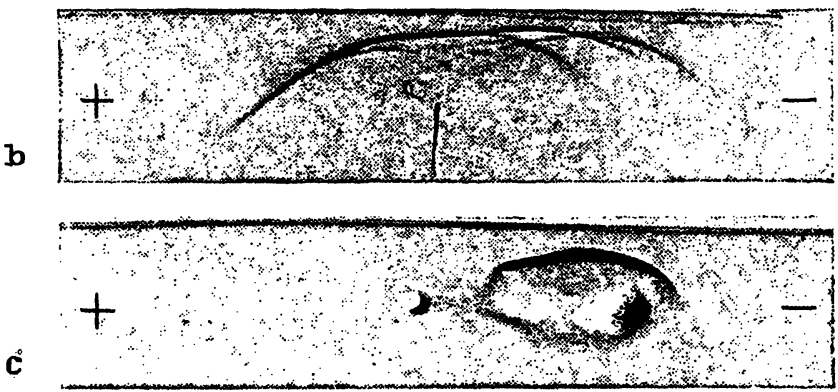

Abb. 2

Immunelektrophorese verschiedener Alkoholdehydrogenasen in 1 proz. Agar, 0,05M Triäthanolamin-Puffer $\mathrm{pH} 7,4$

Im Startloch war: a) und b) Hefealkoholdehydrogenase (HADH), c) Leberalkoholdehydrogenase (LADH)

c) Leber: a) $\mathrm{HADH}-\mathrm{Antiserum}$ Charge A, b) $\mathrm{HADH}$ Antiserum Charge $B, c)$ LADH-Antiserum Einzelheiten s. Text 


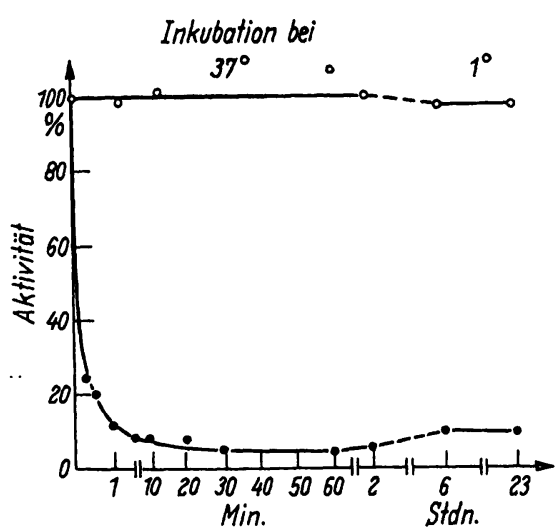

Abb. 3

Hemmung der Aktivität der Hefealkoholdehydrogenase durch ihr spezifisches Antiserum in vitro Testansatz s. Methodik — Aktivität ohne Antiserum

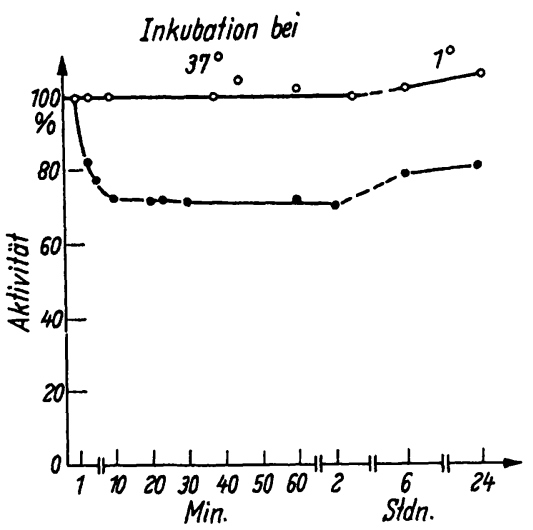

Abb. 4

Hemmung der Aktivität der Alkoholdehydrogenase aus Pferdeleber durch ihr spezifisches Antiserum in vitro

Testansatz s. Methodik

- Aktivität ohne Antiserum Aktivität mit Antiserum

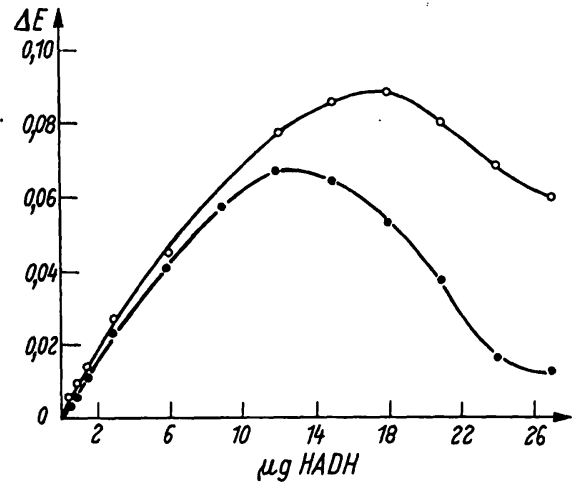

Abb. 5

Nephelometrische Messung des Enzymproteins der Hefealkoholdehydrogenase ( $\mathrm{HADH})$

Gesamtvolumen $2,0 \mathrm{~m} l, 0,2 \mathrm{~m} l \mathrm{HADH}-$ Antiserum Charge $A, d=1 \mathrm{~cm}, 334 \mathrm{~nm}$, $37^{\circ}, 0,015 \mathrm{M} \mathrm{NaCl} 0,002 \mathrm{M}$ an EDTA —- Inkubationszeit $5 \mathrm{Min}$. präzipitierende Antikörper im Kaninchenblutserum nachweisen, jedoch haben diese Seren noch niedrige Titer. 1 Monat nach der ersten Injektion gewonnene Seren mit einem Titer von 4 (Charge A) zeigen im Oucbterlony-Test und in der Immunelektrophorese eine Präzipitationslinie (Abb. 1 u. 2). Geboosterte Seren (Charge B) weisen im Oucbterlony-Test 3 Präzipitationslinien (zwei davon sind bis zur Verdünnung 1:4 sichtbar, Abb. 1), immunelektrophoretisch 4-5 Linien (Abb. 2) auf.

\section{Alkoboldebydrogenase aus Pferdeleber ( $L A D H$ )}

Das nach der angegebenen Methode gewonnene Antiserum mit einem Titer von 8 gibt mit LADH sowohl im Oucbterlony-Test als auch in der Immunelektrophorese eine Präzipitationslinie (Abb. 1 u. 2).

Kreuzreaktionen (HADH-Antiserum Charge A und B gegen $\mathrm{LADH}, \mathrm{LADH}$-Antiserum gegen $\mathrm{HADH}$ ) werden weder immunelektrophoretisch noch im Oucbterlony-Test beobachtet.

Hemmung der Enzymaktivität der HADH und LADH durch spezifische Antiseren

Hefealkoboldebydrogenase ( $H A D H$ )

$0,2 \mathrm{~m} l \mathrm{HADH}$-Antiserum der Charge $\mathrm{A}$ und $\mathrm{B}$ hemmen 0,3-6 $\mu \mathrm{g} \mathrm{HADH}$ innerhalb $30 \mathrm{Min}$. um mehr als $91 \%$ der Ausgangsaktivität (Abb. 3). In den ersten 60 Sek. nach Vereinigung von Enzym und Antiserum sind bereits bis zu 90\% der Ausgangsaktivität gehemmt. Jedoch wird die Enzymaktivität nie vollständig gehemmt, weder in Inkubationsansätzen bis zư $2 \mathrm{Stdn}$. bei $37^{\circ}$ noch bei $1^{\circ}$ nach $23 \mathrm{Stdn}$. Doppelbestimmungen zeigen gut übereinstimmende Werte.

\section{Pferdeleberalkoboldebydrogenase ( $L A D H$ )}

$0,2 \mathrm{~m} l$ spezifisches Antiserum hemmen $10 \mu \mathrm{g} \mathrm{LADH}-$ Enzymprotein um 30\% der Ausgangsaktivität (Abb. 4).
Die doppelte Enzymmenge wird unter den gleichen Bedingungen nur um $10 \%$ gehemmt. Die Zeit, die bis zum Eintritt der größtmöglichen Enzymhemmung benötigt wird, ist etwa $10 \mathrm{mal}$ so lang wie beim $\mathrm{HADH}-V e r-$ such.

\section{Nephelometrische Enzymproteinmessung Eichkurven fïr $H A D H$}

Bei einem konstanten Ansatzvolumen von $2,0 \mathrm{~m} l$ verläuft die Extinktionszunahme bei einer Inkubationsdauer von 5 bzw. 30 Min. zwischen 0,6 und 3,0 $\mu \mathrm{g}$ $\mathrm{HADH}$ als Antigen und $0,2 \mathrm{ml}$ Antiserum Charge A linear und erscheint für quantitative Enzymproteinbestimmungen geeignet (Abb. 5). Größere Mengen von $\mathrm{HADH}$ (Antigen-Überschuß) bewirken eine Verminderung der Extinktion: Die Immunpräzipitate werden nach $5 \mathrm{Min}$. Inkubationsdauer bei $12 \mu \mathrm{g}$ bzw. nach 30 Min. Inkubationsdauer bei $18 \mu \mathrm{g}$ wieder löslicher, jedoch nach verschieden langer Inkubation in unterschiedlicher Weise (Abb. 5).

Ein konzentrierterer Ansatz (0,35 $\mathrm{m} l$ Gesamtvolumen) bringt, wie erwartet, eine bessere Extinktionsausbeute. Die Abhängigkeit von der Inkubationszeit und der Antigenmenge bei konstantem Antiserumvolumen (Antiserum der Charge B) ist in Abbildung 6 zu sehen. Der Extinktionszuwachs ändert sich nach der 50. Inkubationsminute für $0,3-4,5 \mu \mathrm{g} \mathrm{HADH}$ in $0,15 \mathrm{M} \mathrm{NaCl}$ wenig, für $6-9 \mu \mathrm{g}$ HADH-Enzymprotein wird das Extinktionsmaximum bereits nach der 30 . bzw. 40. Inkubationsminute erreicht. Für die Salzkonzentrationen 0,015M und $1,5 \mathrm{M} \mathrm{NaCl}$ werden ähnlich verlaufende Kurven erzielt (vgl. Abb. 10 für LADH), jedoch wird in $1,5 \mathrm{M} \mathrm{NaCl}$ das Extinktionsmaximum bereits nach der 40 . Inkubationsminute für alle eingesetzten Enzymproteinmengen beobachtet. In den Kurven (Abb. 6-11) stellen die jeweiligen Werte Mittelwerte aus 2-4 Einzelmessungen dar. 


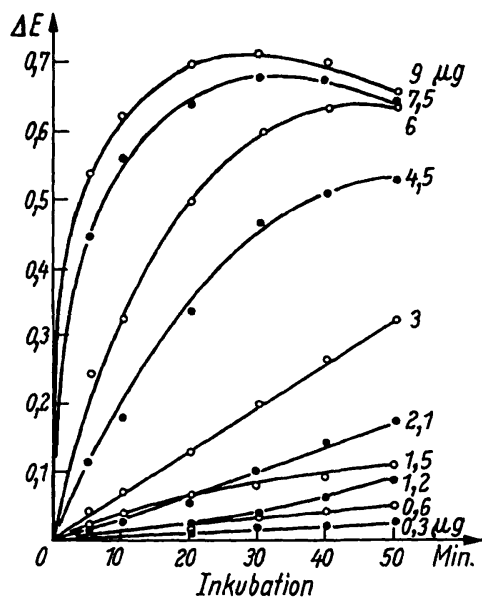

Abb. 6

Nephelometrische Messung des Enzymproteins der Hefealkoholdehydrogenase (HADH) in Abhängigkeit von der Inkubationszeit

Gesamtvolumen $0,35 \mathrm{ml}$. $0,15 \mathrm{M} \mathrm{NaCl}$ $0,2 \mathrm{ml}$ Antiserum Charge $\mathrm{B}, \mathrm{d}=1 \mathrm{~cm}$ $334 \mathrm{~nm}, 25^{\circ}$ (s. Methodik)

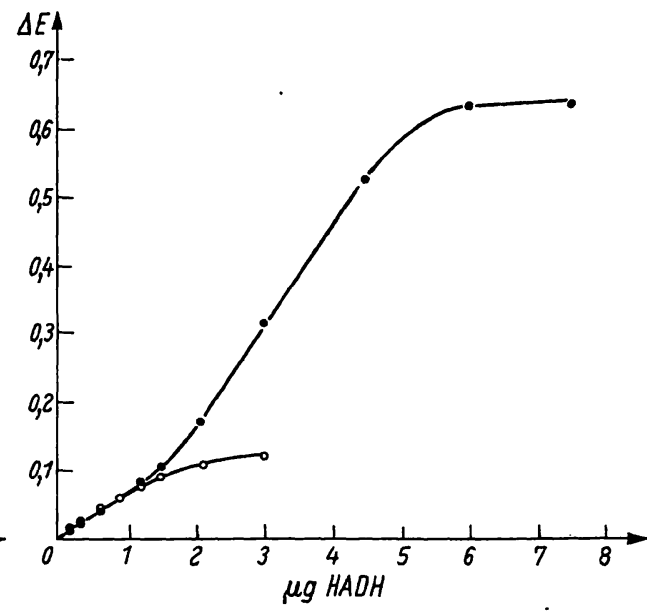

Abb. 7

Nephelometrische Messung des Enzymproteins der Hefealkoholdehydrogenase (HADH)

- - Gesamtvolumen $0,35 \mathrm{ml}, 0,15 \mathrm{M} \mathrm{NaCl}$, $0,2 \mathrm{ml}$ Antiserum Charge B, $334 \mathrm{~nm}$ Gesamtvolumen $0,40 \mathrm{ml}, 0,15 \mathrm{M} \mathrm{NaCl}$, $0,1 \mathrm{ml}$ Antiserum Charge A, $336 \mathrm{~nm}$ $25^{\circ}, \mathrm{d}=1 \mathrm{~cm}$ (s. Methodik)

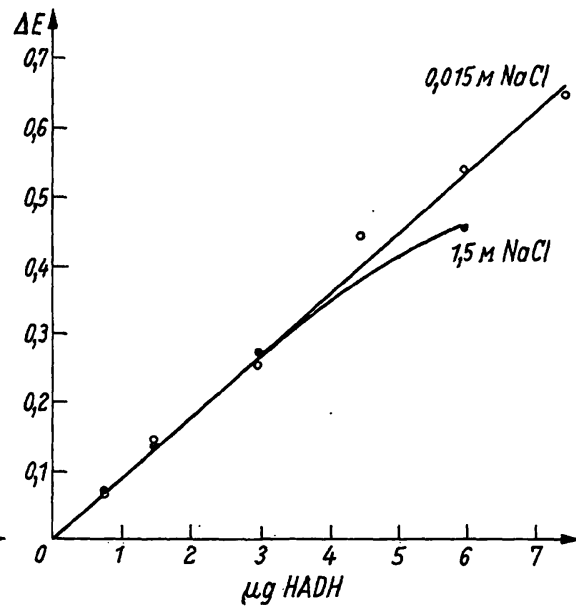

Abb. 8

Nephelometrische Messung des Enzymproteins der Hefealkoholdehydrogenase $(\mathrm{HADH})$ in verschiedenen Salzkonzentrationen

$\stackrel{\longrightarrow}{\longrightarrow} 0,5 \mathrm{M} \mathrm{NaCl}$

Gesamtvolumen 0,35 $\mathrm{ml}, 0,2 \mathrm{ml}$ Antikörper Charge $B, d=1 \mathrm{~cm}, 334 \mathrm{~nm}, 25^{\circ}$ (s.
Zur Aufstellung von Eichkurven werden für 0,15M und $0,015 \mathrm{M} \mathrm{NaCl}$ die Extinktionen der 50. Inkubationsminute gewählt, für $1,5 \mathrm{M} \mathrm{NaCl}$ diejenigen der 40 . Inkubationsminute (Abb. 7 u. 8.).

Die Eichkurve mit $H A D H$-Antiserum Charge $B$ setzt sich in $0,15 \mathrm{M} \mathrm{NaCl}$ reproduzierbar aus zwei Kurven unterscbiedlichen Anstiegs zusammen (Abb. 7): Im Bereich von $0,15 \mu \mathrm{g}$ bis $2 \mu \mathrm{g} \mathrm{HADH}$ hat $1 \mu \mathrm{g}$ die Extinktion $\mathrm{E}_{\mathrm{d}=1 \mathrm{~cm}}^{334 \mathrm{~nm}}=0,076 \pm 0,004, \quad z$ wischen $2 \mu \mathrm{g}$ und $5 \mu \mathrm{g}$ $\mathrm{HADH} \mathrm{E}=0,108 \pm 0,008$. Der Unterschied ist signifikant $(p=0,001, t$-Test). Die durchschnittliche Extinktion für $1 \mu \mathrm{g} \mathrm{HADH}$ errechnet sich hieraus mit $\mathrm{E}_{\mathrm{d}=1 \mathrm{~cm}}^{33 \mathrm{~nm}}=0,089 \pm 0,017$.

Für die Salzkonzentrationen $0,015 \mathrm{M}$ und $1,5 \mathrm{M} \mathrm{NaCl}$ ändert sich diese Extinktion nicht: Sie beträgt für beide $\mathrm{NaCl}$-Konzentrationen $\quad E_{d=1 \mathrm{~cm}}^{334 \mathrm{~nm}}=0,091 \pm 0,008$ pro $1 \mu \mathrm{g} \mathrm{HADH}$, nach der 50. bzw. 40. Inkubationsminute gemessen. Im Gegensatz zur 0,15 $\mathrm{M} \mathrm{NaCl}$-Eichkurve verlaufen diejenigen der $0,015 \mathrm{M}$ bzw. 1,5M NaCl-Konzentrationen linear (Abb. 8).

Im Gegensatz zum HADH-Antiserum (Charge B) zeigt die Eichkurve mit $H A D H$-Antiserum Charge $A$ in $0,15 \mathrm{M} \mathrm{NaCl}$ einen linearen Kurvenverlauf zwischen 0,15 und $1 \mu \mathrm{g} \mathrm{HADH}$-Enzymprotein, eine zusammengesetzte Kurve ist nicht zu sehen (Abb. 7). Die Extinktion für $1 \mu \mathrm{g} \mathrm{HADH}$ beträgt $\mathrm{E}_{\mathrm{d}=1 \mathrm{~cm}}^{366 \mathrm{~nm}}=0,080 \pm 0,007$ für die 50. Inkubationsimnute und $\mathrm{E}=0,090 \pm 0,009$ für die 70. Minute.

\section{Eicbleurven für $L A D H$}

Die Abhängigkeit der Extinktion von Inkubationszeit, Antigenmenge und Salzkonzentration bei konstantem Antiserumvolumen ist in den Abbildungen 9 und $10 \mathrm{zu}$ sehen. Das Extinktionsmaximum wird für die einge- setzten Enzymproteinmengen in $0,15 \mathrm{M}$ und $0,015 \mathrm{M} \mathrm{NaCl}$ nach der 40 . Inkubationsminute erreicht, in $1,5 \mathrm{M} \mathrm{NaCl}$ für höhere Konzentrationen an $\mathrm{LADH}$ bereits nach der 20. bis 30. Inkubationsminute (Abb. 10). Die Extinktion für $1 \mu \mathrm{g} \mathrm{LADH}$ beträgt in $0,15 \mathrm{M} \mathrm{NaCl} \mathrm{E} \mathrm{E}_{\mathrm{d}=1 \mathrm{~cm}}^{334 \mathrm{~nm}}$ $=0,127 \pm 0,003$, in $0,015 \mathrm{M} \mathrm{NaCl} \mathrm{E} \mathrm{d}_{\mathrm{d}=1 \mathrm{~cm}}^{334 \mathrm{~nm}}=0,179 \pm$ 0,008 und in $1,5 \mathrm{M} \mathrm{NaCl} \mathrm{E} \mathrm{E}_{\mathrm{d}=1 \mathrm{~cm}}^{334 \mathrm{~mm}}=0,150 \pm 0,008$ nach der 40. Inkubationsminute (Abb. 11). Die mittleren Extinktionswerte für $1 \mu \mathrm{g} \mathrm{LADH}$ in den $3 \mathrm{NaCl}$ Konzentrationen unterscheiden sich untereinander signifikant $(p<0,001, t-$ Test $)$.

\section{Messung von $H A D H$-Enz ymprotein im Hefehomogenat}

In einem in 0,5M Glycyl-Glycin-Puffer $\mathrm{pH} 7,4$ extrahierten und bei 3000 U./Min. abzentrifugierten Hefehomogenat mit einer HADH-Aktivität von 28 IU $\left(37^{\circ}\right) / \mathrm{mg}$ Biuretprotein (entsprechend 133,4 $\mu \mathrm{g}$ Enzymprotein des Eichpräparates, rechnerisch ermittelt) werden $143,2 \mu \mathrm{g}$ HADH-Enzymprotein nephelometrisch gemessen (Tab. 1). Hierzu werden allerdings längere Inkubationszeiten erforderlich.

\section{Messung von $H A D H$-Enzymprotein in Serum}

In Gegenwart eines Uberschusses an Fremdprotein werden mit Hilfe der nephelometrischen EnzymproteinMessung 0,3 $\mu \mathrm{g}$ und $0,6 \mu \mathrm{g} \mathrm{HADH}$ mit guter Ausbeute und einem mittleren Fehler von $\pm 4 \%$ wiedergefunden. (Tab. 2). Die Enzymaktivität der HADH bleibt in Gegenwart von Serum vom Menșchen unbeeinflußt.

\section{Einfluß der Wellenlänge auf die Extinktionsausbeute}

Das Messen der Trübung bei anderen Wellenlängen ergibt eine deutliche Verminderung der Ausbeute: Setzt man die bei 334 nm gemessene Extinktion gleich 100\%, 


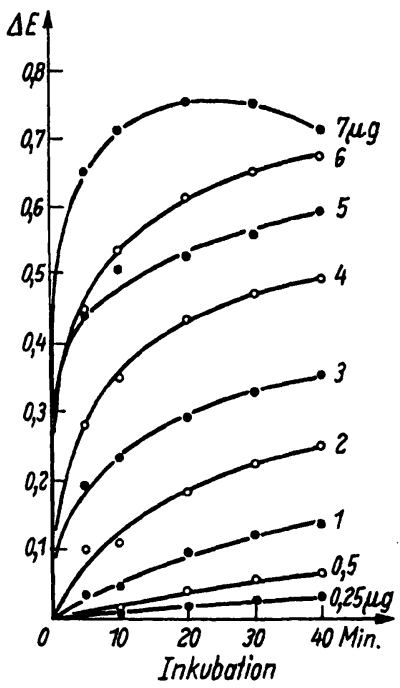

Abb. 9

Nephelometrische Messung des Enzymproteins derLeberalkoholdehydrogenase (LADH) in Abhängigkeit von der Inkubationszeit und der $\mathrm{NaCl}-\mathrm{Konzentration}$ Gesamtvolumen $0,35 \mathrm{ml}, 0,15 \mathrm{M}$ $\mathrm{NaCl}, 0,2 \mathrm{ml}$ Antiserum, $d=1 \mathrm{~cm}$ $334 \mathrm{~nm}, 25^{\circ}$ (s. Methodik)

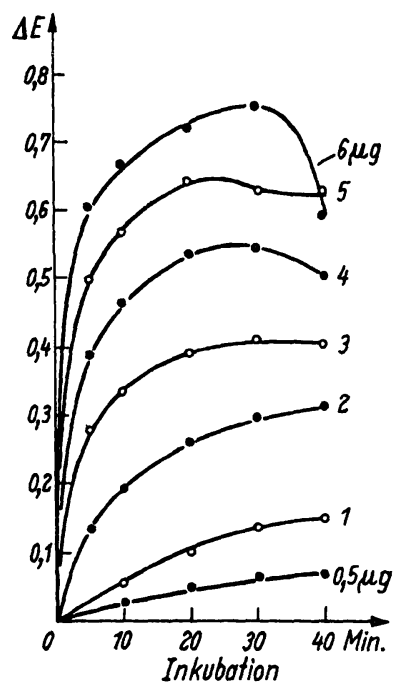

Abb. 10

Nephelometrische Messung des Enzymproteins derLeberalkoholdehydrogenase (LADH) in Abhängigkeit von der Inkubationszeit und der $\mathrm{NaCl}$-Konzentration Gesamitvolumen $0,35 \mathrm{ml}, 1,5 \mathrm{M} \mathrm{NaCl}$, $0,2 \mathrm{ml}$ Antiserum, $\mathrm{d}=1 \mathrm{~cm}, 334 \mathrm{~nm}$, $25^{\circ}$ (s. Methodik)

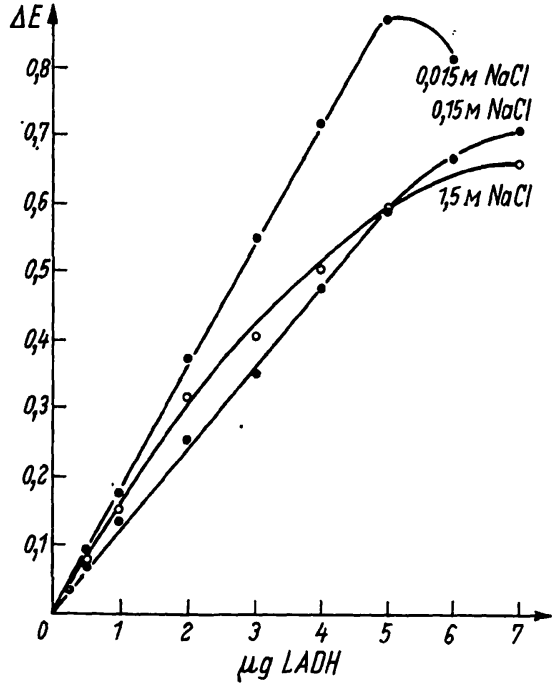

Abb. 11

Nephelometrische Messung des Enzym. proteins der Leberalkoholdehydrogenase $(\mathrm{LADH})$ in verschiedenen $\mathrm{NaCl}-\mathrm{Konzen}$ trationen

Gesamtvolumen $0,35 \mathrm{ml}, 0,2 \mathrm{ml}$ Antiserum, $\mathrm{d}=1 \mathrm{~cm}, 334 \mathrm{~nm}, 25^{\circ}$ (s. Text)

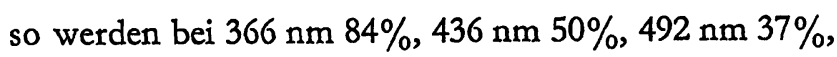
$546 \mathrm{~nm} 35 \%$ und bei $578 \mathrm{~nm} 32 \%$ dieses Wertes gemessen.

\section{Diskussion}

Die Hemmung der Aktivität der Alkoholdehydrogenase aus Hefe (HADH) durch spezifische Antikörper ist bereits bekannt (16-19). Obwohl bei Antikörperüberschuß bereits 20 Sek. nach der Vereinigung von $\mathrm{HADH}$ mit dem spezifischen Antikörper mehr als 70\% ihrer Aktivität gehemmt wird, scheint die Bindung des Antikörpers nicht an den aktiven Zentren des Enzyms stattzufinden (18).

Die Aktivität der Alkoholdehydrogenase aus Pferdeleber (LADH) wird durch spezifische Antikörper weit weniger gehemmt. Es erscheint uns deshalb unangebracht, mit Hilfe der Entaktivierung von Enzymaktivitäten durch spezifische Antikörper einen Test zur Messung des Enzymproteins aufzubauen.

Die Messung von Proteinen im Blutserum mit Hilfe ihres präzipitierenden Antigen-Antikörper-Komplexes ist seit langem bekannt $(5,6,20-29)$ und mit der verbesserten Darstellung von Reinproteinen vervollkomm net worden $(7-9,24)$.

Die gute Ubereinstimmung bei diesen Untersuchungen zwischen dem nach HeIDELberger und Kendali bestimmten N-Gehalt im Präzipitat mit der nephelometrisch gemessenen Antikörper-Antigen-Menge $(8,9)$ veranlaßten uns, die bei der Vereinigung von Enzymprotein und Antikörper entstehende Trübung im Photometer Eppendorf in einem Serien-Mikro-Test zur Erfassung von Enzymproteinen der HADH und LADH aufzubauen.
Bei der Aufstellung von Eichkurven für HADH-Enzymprotein ergeben zwei verschiedene Antiseren (ein nicht geboostertes mit niedrigem Titer und ein geboostertes mit hohem Titer) unterscbiedliche Eichkurven: Beide Seren zeigen in einem Bereich zwischen 0,15 $\mu \mathrm{g}$ und 1,0 $\mu \mathrm{g}$ Enzymprotein identische Extinktionen und einen linearen Kurvenverlauf, bei höheren Enzymkonzentrationen steigt die mit dem geboosterten Serum erlangte Eichkurve steil an, während diejenige mit dem nicbt geboosterten Antiserum relativ flach verläuft (Abb. 7). Die mit dem geboosterten Antiserum gewonnene Eichkurve setzt sich reproduzierbar aus zwei Kurven unterschiedlichen Anstiegs zusammen. Da dieses Serum im Ouchterlony-Test und in der Immunelektrophorese gegenüber $\mathrm{HADH} 3$ bzw. 4 verschiedene, nicht ineinander übergehende Präzipationslinien zeigt, was entweder auf Isoenzyme der HADH $(30,31,33)$ oder aber auf Verunreinigungen des Enzympräparates, die beim Boostern besonders stark herauskommen, zurückgeführt werden kann, ließe sich die aus zwei Kurven zusammengesetzte HADH-Eichkurve in $0,15 \mathrm{M} \mathrm{NaCl}$ insofern hierauf $z u-$ rückführen, als bei niedrigeren Konzentrationen an HADH sich jene Störfaktoren infolge ihrer viel geringeren Menge nicht bemerkbar machen würden. Dieses Phänomen der zusammengesetzten Eichkurve ist jedoch abhängig von der $\mathrm{NaCl}$-Konzentration, in der die Antigen-Antikörperreaktion stattfindet: In $0,015 \mathrm{M}$ und $1,5 \mathrm{M} \mathrm{NaCl}$ werden nur einfach-lineare Eichkurven für $\mathrm{HADH}$ gefunden.

Aus den Untersuchungen von NAJJAR und FISHER ist bekannt, daß Kaninchen Antikörper auf HADH-Antikörper-Komplexe bei längeren Immunisierungszeiten bilden. Da verschiedene Salzkonzentrationen jeweils be- 
stimmte Antigen-Antikörper-Komplexe zum Ausfällen bringen (32, 2-4), nehmen wir an, daß die in $0,15 \mathrm{M} \mathrm{NaCl}$ gefundene, zusammengesetzte Eichkurve auf den'sich bei höheren Antigen-Antikörper-Konzentrationen bemerkbar machenden (Antigen-Antikörper)-AntikörperKomplex zurückzuführen ist, der in $0,015 \mathrm{M}$ bzw. in $1,5 \mathrm{M}$ $\mathrm{NaCl}$ nicht in dem gleichen Ausmaß gebildet werden dürfte.

Auch die bei der LADH-Antikörperreaktion beobachteten, in verschiedenen $\mathrm{NaCl}$-Konzentrationen unterschiedlichen Eichkurven dürften auf verschiedenartige Löslichkeit des Antigen-Antikörperkomplexes und möglicherweise auch auf Kopräzipitation zurückzuführen sein.

Um diese möglichen Störreaktionen auszuschalten, haben wir einen Test zur Messung von HADH-Enzym- protein mit einem ungeboosterten Antiserum aufgebaut und das HADH-Enzymprotein im Hefehomogenat bzw. in Gegenwart von viel Fremdprotein gemessen.

Im Hefehomogenat stimmt die aus der Enzymaktivität berechnete Menge an Enzymprotein gut mit der nephelometrisch gefundenen überein. Durch längere Inkubationszeiten (bis $70 \mathrm{Min}$.) kann die durch Kopräzipitation verursachte Trübung eliminiert werden. In Gegenwart von viel Fremdprotein (Blutserum vom Menschen) wird das eingesetzte HADH-Enzymprotein mit einem mittleren Fehler von $\pm 4 \%$ wiedergefunden.

Herrn Prof. Dr. H. Hrzz danke ich für wertvolle Anregung, Beratung und Unterstützung bei der Durchführung dieser Arbeit sowie Herrn Dr. H. Eckstern für die Überlassung des Hefehomogenates.

\section{Literatur}

1. Hess, B., Enzyme im Blutplasma, Georg Thieme Verlag, Stuttgatt (1962). - 2. Goodman, M., H. R. Wolfe und S. Norton, J. Immunology 66, 225 (1951). - 3. Goodman, M. und H. R. Wolfe, J. Immunology 69, 423 (1952). - 4. Goodman, M., H. R. Wolfe und R. Goldberg, J. Immunology 72, 440 (1954). 5. Goodman, M., D. S. Ramsey, W. L. Simpson, D. G. Remps, D. H. Basinski und M. J. Brennan, J. Laborat. Clin. Med., S. Louis 49, 151 (1957). - 6. Goodman, M., D. S. Ramsey, E. L. Simpson und M. J. Brennan, J. Laborat. Clin. Med., S. Louis 50, 758 (1957). - 7. Goodman, M., H. S. Newman und D. S. Ramsey, J. Laborat. Clin. Med., S. Louis 51, 814 (1958). - 8. ScheIfFarth, F., H. Götz und H. Warnatz, Clin. chim. Acta (Amsterdam) 3, 535 (1958). - 9. Schultze, H. E. und G. Schwrck, Clin. chim. Acta (Amsterdam) 4, 15 (1959). - 10. OuchterLoNY, Ö., GelDiffusion Techniques in Immunological Methods. Blackwell Scientific Publ., Oxford (1964). - 11. Grabar, P. und P. Burtin, Immuno-Elektrophoretische Analyse. Elsevier Publ. Comp., Amsterdam (1964). - 12. Scheidegger, J. J., Internat. Arch. Allergy 7, 103 (1955). - 13. RACKER, E., in: Methods in Enzymology Bd. I, S. 500. Academic Press, Nerv York (1955). - 14. BoNNICHSEN, R. K. und N. G. BRINK, in: Methods in Enzymology, Hrsg. S. P. Colowick und N. O. Kaplan. Bd. I, S. 495. Academic Press, New York (1955). - 15. NefF, J. C. und E. L. BeCker,
J. Immunology 73, 286 (1954). - 16. JohanNSMEIER, K. und H. ReDeTzkr, Biochem. Z. 326, 515 (1955). - 17. NajJaR, V. A. und J. Fisher, Biochim. biophysica Acta (Amsterdam) 20, 158 (1956). - 18. Pfleiderer; G., Z. Kopifar und D. E. Wachsmuth, Biochem. Z. 341, 239 (1965). - 19. Redetzkr, H. M., Proc. Soc. exp. Biol. Med. 98, 120 (1958). - Boyden, A., E. Bolten und D. Gemeroy, J. Immunology 57, 211 (1947). - 21. EISEN, H. N., J. Immunology 60, 77 (1948). - 22. GirLIn, D., J. Immunology 62, 437 (1949). - 23. Grimr, D. und H. Epelhoch, J. Immunology 66, 67 (1951). - 24. Haferkamp, O., D. SchletwernGsell, H. G. Schwick und K. StörIKo, Klin. Wschr. 44, 725 (1966). - 25. HAYDEN, A. R. und E. L. BECKER, J. Immunology 85, 591 (1960). - 26. RuBINSTEIn, H. M., J. Immunology 73, 322 (1954). - 27. Schмrdт, W. C., J. Immunology 78, 178 (1957). 28. Tengerdy, R. P., Analyt. Biochem. (New York) 11, 272 (1965). -29. Tengerdy, R. P. und W. H. Small, Nature (London) 210, 708 (1966). - 30. Watts, D. C., C. Dominger und E. P. WhiteHEAD, Biochem. J. 81, 4p (1961). - 31. WATTS, D. C., und C. Dominger, Analyt. Biochem. (New York) 3, 489 (1962). - 32. ALADJEM, F. und M. LIEBERMAN, J. Immunology 69, 117 (1952). 33. Papenderg, J., J. P. v. Wartburg und H. Aebi, Biochem. $Z$. 342, 95 (1965).

Dr. T. O. Kleine

2 Hamburg 20, Martinistr. 52 